\title{
Finite-difference method for the hyperbolic system of equations with nonlocal boundary conditions
}

\author{
Allaberen Ashyralyev ${ }^{1,2^{*}}$ and Rahat Prenov ${ }^{2}$
}

\author{
"Correspondence: \\ aashyr@fatih.edu.tr \\ ${ }^{1}$ Department of Mathematics, Fatih \\ University, Istanbul, Turkey \\ ${ }^{2}$ Department of Mathematics, ITTU, \\ Ashgabat, Turkmenistan
}

\begin{abstract}
In the present paper, the finite-difference method for the initial-boundary value problem for a hyperbolic system of equations with nonlocal boundary conditions is studied. The positivity of the difference analogy of the space operator generated by this problem in the space $C$ with maximum norm is established. The structure of the interpolation spaces generated by this difference operator is investigated. The positivity of this difference operator in Hölder spaces is established. In applications, stability estimates for the solution of the difference scheme for a hyperbolic system of equations with nonlocal boundary conditions are obtained. A numerical example is applied.
\end{abstract}

MSC: $35\llcorner 40 ; 35 L 45$

Keywords: hyperbolic system of equations; nonlocal boundary value problems; difference schemes; interpolation spaces; positivity of the difference operator; stability estimates

\section{Introduction}

Nonlocal problems are widely used for mathematical modeling of various processes of physics, ecology, chemistry, and industry, when it is impossible to determine the boundary or initial values of the unknown function. The method of operators as a tool for the investigation of the solution of local and nonlocal problems for partial differential equations in Hilbert and Banach spaces has been systematically developed by several authors (see, e.g., [1-27]). It is well known that (see, e.g., [28-32] and the references given therein) many application problems in fluid mechanics, physics, mathematical biology, and chemistry were formulated as nonlocal mathematical models. Note that such problems were not well studied in general.

In the paper [33], the initial-boundary value problem

$$
\left\{\begin{array}{l}
\frac{\partial u(t, x)}{\partial t}+a(x) \frac{\partial u(t, x)}{\partial x}+\delta(u(t, x)-v(t, x))=f_{1}(t, x), \quad 0<x<l, 0<t<T, \\
\frac{\partial v(t, x)}{\partial t}-a(x) \frac{\partial v(t, x)}{\partial x}+\delta v(t, x)=f_{2}(t, x), \quad 0<x<l, 0<t<T, \\
u(t, 0)=\gamma u(t, l), \quad 0 \leq \gamma \leq 1, \quad \beta v(t, 0)=v(t, l), \quad 0 \leq \beta \leq 1,0 \leq t \leq T, \\
u(0, x)=u_{0}(x), \quad v(0, x)=v_{0}(x), \quad 0 \leq x \leq l
\end{array}\right.
$$

\section{Springer}

(2014 Ashyralyev and Prenov; licensee Springer. This is an Open Access article distributed under the terms of the Creative Commons Attribution License (http://creativecommons.org/licenses/by/2.0), which permits unrestricted use, distribution, and reproduction in any medium, provided the original work is properly cited. 
for the hyperbolic system of equations with nonlocal boundary conditions was considered. Here

$$
a(x) \geq a>0,
$$

$u_{0}(x), v_{0}(x)(x \in[0, l]), f_{1}(t, x), f_{2}(t, x)((t, x) \in[0, T] \times[0, l])$ are given smooth functions and they satisfy all compatibility conditions which guarantee the problem (1) has a smooth solution $u(t, x)$ and $v(t, x)$. As noted in the paper [33], the problem of sound waves [34] and the problem of the expansion of electricity oscillations [35] can be replaced by the problem (1). Note that, we have the nonclassical initial-boundary value problem (1) with boundary conditions $u(t, 0)=\gamma u(t, l), 0 \leq \gamma \leq 1, \beta v(t, 0)=v(t, l), 0 \leq \beta \leq 1,0 \leq t \leq T$. These conditions are given on two boundary points. It is clear that it is impossible to determine the boundary values of the unknown function. So, these conditions are not local.

Let $E$ be a Banach space and $A: D(A) \subset E \rightarrow E$ be a linear unbounded operator densely defined in $E$. We call $A$ a positive operator in the Banach space if the operator $(\lambda I+A)$ has a bounded inverse in $E$ for any $\lambda \geq 0$, and the following estimate holds:

$$
\left\|(\lambda I+A)^{-1}\right\|_{E \rightarrow E} \leq \frac{M}{\lambda+1} .
$$

Throughout the present paper, $M$ is defined as a positive constant. However, we will use $M(\alpha, \beta, \ldots)$ to stress the fact that the constant depends only on $\alpha, \beta, \ldots$

For a positive operator $A$ in the Banach space $E$, let us introduce the fractional spaces $E_{\alpha}=E_{\alpha}(E, A)(0<\beta<1)$ consisting of those $v \in E$ for which the norm

$$
\|v\|_{E_{\alpha}}=\sup _{\lambda>0} \lambda^{\alpha}\left\|A(\lambda+A)^{-1} v\right\|_{E}+\|v\|_{E}
$$

is finite.

Let us introduce the Banach space $\mathbb{C}^{\alpha}[0, l]=C^{\alpha}([0, l], R) \times C^{\alpha}([0, l], R)(0 \leq \alpha \leq 1)$ of all continuous vector functions $u=\left(\begin{array}{c}u_{1}(x) \\ u_{2}(x)\end{array}\right)$ defined on $[0, l]$ and satisfying a Hölder condition for which the following norm is finite:

$$
\begin{aligned}
\|u\|_{\mathbb{C}^{\alpha}[0, l]}= & \|u\|_{\mathbb{C}[0, l]} \\
& +\sup _{\substack{x, x+\tau \in[0, l] \\
\tau \neq 0}} \frac{\left|u_{1}(x+\tau)-u_{1}(x)\right|}{|\tau|^{\alpha}}+\sup _{\substack{x, x+\tau \in[0, l] \\
\tau \neq 0}} \frac{\left|u_{2}(x+\tau)-u_{2}(x)\right|}{|\tau|^{\alpha}} .
\end{aligned}
$$

Here $\mathbb{C}[0, l]=C([0, l], R) \times C([0, l], R)$ is the Banach space of all continuous vector functions $u=\left(\begin{array}{l}u_{1}(x) \\ u_{2}(x)\end{array}\right)$ defined on $[0, l]$ with norm

$$
\|u\|_{\mathbb{C}[0, l]}=\max _{x \in[0, l]}\left|u_{1}(x)\right|+\max _{x \in[0, l]}\left|u_{2}(x)\right|
$$

We consider the space operator $A$ generated by the problem (1) defined by the formula

$$
A u=\left(\begin{array}{cc}
a(x) \frac{d u_{1}(x)}{d x}+\delta u_{1}(x) & -\delta u_{2}(x) \\
0 & -a(x) \frac{d u_{2}(x)}{d x}+\delta u_{2}(x)
\end{array}\right)
$$


with domain

$$
\begin{aligned}
D(A)= & \left\{\left(\begin{array}{l}
u_{1}(x) \\
u_{2}(x)
\end{array}\right): u_{m}(x), \frac{d u_{m}(x)}{d x} \in C([0, l], R), m=1,2 ;\right. \\
& \left.u_{1}(0)=\gamma u_{1}(l), \beta u_{2}(0)=u_{2}(l)\right\} .
\end{aligned}
$$

The Green's matrix function of $A$ was constructed. The positivity of the operator $A$ in the Banach space $\mathbb{C}[0, l]$ was established. It was proved that for any $\alpha \in(0,1)$ the norms in spaces $E_{\alpha}(\mathbb{C}[0, l], A)$ and $\stackrel{\circ}{\mathbb{C}}^{\alpha}[0, l]$ are equivalent. The positivity of $A$ in the Hölder spaces of $\stackrel{\circ}{\mathbb{C}}^{\alpha}[0, l], \alpha \in(0,1)$ was proved. In applications, stability estimates for the solution of the problem (1) for the hyperbolic system of equations with nonlocal boundary conditions were obtained.

In the present paper, the finite-difference method for the initial value problem for the hyperbolic system of equations with nonlocal boundary conditions is applied. The positivity of the difference analogy of the space operator $A$ defined by equation (1) in the difference analogy of $\mathbb{C}[0, l]$ spaces is established. The structure interpolation spaces generated by this difference operator is studied. The positivity of this difference operator in Hölder spaces is established. In practice, stability estimates for the solution of the difference scheme for the hyperbolic system of equations with nonlocal boundary conditions are obtained. The method is illustrated by numerical example.

The organization of the present paper as follows. Section 1 is an introduction where we provide the history and formulation of the problem. In Section 2, the Green's matrix function of the difference space operator is presented and positivity of this operator in the difference analogy of $\mathbb{C}[0, l]$ spaces is proved. In Section 3, the structure of fractional spaces generated by this difference operator is investigated and positivity of this difference operator in Hölder spaces is established. In Section 4, stable difference schemes for the approximate solution of the problem (1) are constructed. A theorem on the stability for the first order of accuracy in the $t$ difference scheme is proved. In Section 5 , a numerical application is given. Finally, Section 6 is for our conclusion.

\section{The Green's matrix function of difference space operator and positivity}

Let us introduce the Banach spaces $\mathbb{C}_{h}^{\alpha}=C_{h}^{\alpha} \times C_{h}^{\alpha}(0 \leq \alpha \leq 1)$ and $\mathbb{C}_{h}=C_{h} \times C_{h}$ of all mesh vector functions $u^{h}=\left\{\left(\begin{array}{c}u_{1, n} \\ u_{2, n-1}\end{array}\right)\right\}_{n=1}^{M}$ defined on

$$
[0, l]_{h}=\left\{x_{n}=n h, 0 \leq n \leq M, M h=l\right\}
$$

with the following norms:

$$
\begin{aligned}
\left\|u^{h}\right\|_{\mathbb{C}_{h}^{\alpha}}= & \left\|u^{h}\right\|_{\mathbb{C}_{h}} \\
& +\sup _{1 \leq n<n+m \leq M} \frac{\left|u_{1, n+m}-u_{1, n}\right|}{(m h)^{\alpha}}+\sup _{1 \leq k<k+m \leq M-1} \frac{\left|u_{2, n+m}-u_{2, n}\right|}{(m h)^{\alpha}}, \\
\left\|u^{h}\right\|_{\mathbb{C}_{h}}= & \max _{1 \leq n \leq M}\left|u_{1, n}\right|+\max _{0 \leq n \leq M-1}\left|u_{2, n}\right| .
\end{aligned}
$$


We consider the difference space operator $A_{h}^{x}$ generated by the problem (1) defined by the formula

$$
A_{h}^{x} u^{h}=\left(\begin{array}{cc}
a\left(x_{n}\right) \frac{u_{1, n}-u_{1, n-1}}{h}+\delta u_{1, n} & -\delta u_{2, n} \\
0 & -a\left(x_{n}\right) \frac{u_{2, n+1}-u_{2, n}}{h}+\delta u_{2, n}
\end{array}\right)
$$

acting on the space of mesh vector functions $u^{h}=\left\{\left(\begin{array}{c}u_{1, n} \\ u_{2, n-1}\end{array}\right)\right\}_{n=1}^{M}$ defined on $[0, l]_{h}$, satisfying the conditions

$$
u_{1,0}=\gamma u_{1, M}, \quad \beta u_{2,0}=u_{2, M} .
$$

Here $a_{n}=a\left(x_{n}\right)$. We will study the resolvent of the difference space operator $-A_{h}^{x}$, i.e.

$$
A_{h}^{x}\left(\begin{array}{l}
u \\
v
\end{array}\right)^{h}+\lambda\left(\begin{array}{l}
u \\
v
\end{array}\right)^{h}=\left(\begin{array}{l}
\varphi \\
\psi
\end{array}\right)^{h}
$$

or

$$
\left\{\begin{array}{l}
a_{n} \frac{u_{n}-u_{n-1}}{h}+(\delta+\lambda) u_{n}-\delta v_{n}=\varphi_{n}, \quad 1 \leq n \leq M, \\
-a_{n+1} \frac{v_{n+1}-v_{n}}{h}+(\delta+\lambda) v_{n}=\psi_{n}, \quad 0 \leq n \leq M-1, \\
u_{0}=\gamma u_{M}, \quad \beta v_{0}=v_{M} .
\end{array}\right.
$$

Lemma 2.1 For any $\lambda \geq 0$, equation (7) is uniquely solvable and the following formula holds:

$$
\begin{aligned}
\left(\begin{array}{l}
u_{n} \\
v_{n}
\end{array}\right) & =\left(A_{h}^{x}+\lambda\right)^{-1}\left(\begin{array}{c}
\varphi_{n} \\
\psi_{n}
\end{array}\right)=\sum_{s=0}^{M} G(n, s ; \lambda)\left(\begin{array}{c}
\varphi_{s} \\
\psi_{s}
\end{array}\right) h \\
& =\left(\begin{array}{c}
\sum_{n=1}^{M} G_{11}(n, s ; \lambda) \varphi_{s} h+\sum_{n=0}^{M-1} G_{12}(n, s ; \lambda) \psi_{s} h \\
\sum_{n=0}^{M-1} G_{22}(n, s ; \lambda) \psi_{s} h,
\end{array}\right), \quad 0 \leq n \leq M,
\end{aligned}
$$

where

$$
G(n, s ; \lambda)=\left(\begin{array}{cc}
G_{11}(n, s ; \lambda) & G_{12}(n, s ; \lambda) \\
0 & G_{22}(n, s ; \lambda)
\end{array}\right) .
$$

Here

$$
\begin{aligned}
& G_{11}(n, s ; \lambda)=Q \begin{cases}U(n, s-1, \lambda) \frac{1}{a_{s}}, & 1 \leq s \leq n, \\
\gamma U(n, 0, \lambda) U(M, s-1, \lambda) \frac{1}{a_{s}}, & n+1 \leq s \leq M,\end{cases} \\
& G_{22}(n, s ; \lambda)=P \begin{cases}\frac{\beta}{a_{s+1}} U(M, n, \lambda) U(s+1,0, \lambda), & 0 \leq s \leq n-1, \\
\frac{1}{a_{s+1}} U(s+1, n, \lambda), & n \leq s \leq M-1,\end{cases} \\
& G_{12}(n, s ; \lambda)=\delta \sum_{k=1}^{M} G_{11}(n, k-1 ; \lambda) G_{22}(k, s ; \lambda) h, \\
& P=(1-\beta U(M, 0 ; \lambda))^{-1}, \quad Q=(1-\gamma U(M, 0 ; \lambda))^{-1},
\end{aligned}
$$




$$
\begin{aligned}
& U(n, k ; \lambda)= \begin{cases}R_{n}, \ldots, R_{k+1}, & n>k, \\
1, & n=k,\end{cases} \\
& R_{n}=\left(1+\frac{(\delta+\lambda) h}{a_{n}}\right)^{-1}, \quad 1 \leq n \leq M .
\end{aligned}
$$

Proof Using the resolvent equation (7), we get

$$
-a_{n+1} \frac{v_{n+1}-v_{n}}{h}+(\delta+\lambda) v_{n}=\psi_{n}, \quad 0 \leq n \leq M-1, \quad \beta v_{0}=v_{M}
$$

From that follows the following recursive formula:

$$
v_{n}=R_{n+1} v_{n+1}+\frac{h}{a_{n+1}} R_{n+1} \psi_{n}, \quad 0 \leq n \leq M-1 .
$$

Hence

$$
v_{n}=U(M, n ; \lambda) v_{M}+\sum_{s=n}^{M-1} U(s+1, n ; \lambda) \frac{h}{a_{s+1}} \psi_{s}, \quad 0 \leq n \leq M-1 .
$$

From this formula and the nonlocal boundary condition $\beta v_{0}=v_{M}$ it follows that

$$
v_{M}=\beta P \sum_{s=0}^{M-1} U(s+1,0 ; \lambda) \frac{h}{a_{s+1}} \psi_{s} .
$$

Then,

$$
\begin{aligned}
v_{n}= & U(M, n ; \lambda) \beta P \sum_{s=0}^{M-1} U(s+1,0 ; \lambda) \frac{h}{a_{s+1}} \psi_{s} \\
& +\sum_{s=n}^{M-1} U(s+1, n ; \lambda) \frac{h}{a_{s+1}} \psi_{s}=\beta P \sum_{s=0}^{n-1} U(M, n ; \lambda) U(s+1,0 ; \lambda) \frac{h}{a_{s+1}} \psi_{s} \\
& +P \sum_{s=n}^{M-1} U(s+1, n ; \lambda) \frac{h}{a_{s+1}} \psi_{s}=\sum_{s=0}^{M-1} G_{22}(n, s ; \lambda) \psi_{s} h .
\end{aligned}
$$

Using the resolvent equation (7), we get

$$
a_{n} \frac{u_{n}-u_{n-1}}{h}+(\delta+\lambda) u_{n}-\delta v_{n}=\varphi_{n}, \quad 1 \leq n \leq M, \quad u_{0}=\gamma u_{M}
$$

From that follows the system of recursion formulas

$$
u_{n}=R_{n} u_{n-1}+\frac{h}{a_{n}} R_{n}\left(\delta v_{n}+\varphi_{n}\right), \quad 1 \leq n \leq M .
$$

Hence

$$
u_{n}=U(n, 0 ; \lambda) u_{0}+\sum_{k=1}^{n} U(n, k-1 ; \lambda) \frac{h}{a_{k}}\left(\delta v_{k}+\varphi_{k}\right), \quad 1 \leq n \leq M
$$


From this formula and the nonlocal boundary condition $u_{0}=\gamma u_{M}$ it follows that

$$
u_{0}=\gamma Q \sum_{k=1}^{M} U(M, k-1 ; \lambda) \frac{h}{a_{k}}\left(\delta v_{k}+\varphi_{k}\right)
$$

Therefore,

$$
\begin{aligned}
u_{n} & =U(n, 0 ; \lambda)\left[\gamma Q \sum_{k=1}^{M} U(M, k-1 ; \lambda) \frac{h}{a_{k}}\left(\delta v_{k}+\varphi_{k}\right)\right]+\sum_{k=1}^{n} U(n, k-1 ; \lambda) \frac{h}{a_{k}}\left(\delta v_{k}+\varphi_{k}\right) \\
& =U(n, 0 ; \lambda) \gamma Q \sum_{s=n+1}^{M} U(M, s-1 ; \lambda) \frac{h}{a_{s}}\left(\delta v_{s}+\varphi_{s}\right)+\sum_{s=1}^{n} U(n, s-1 ; \lambda) \frac{h}{a_{s}}\left(\delta v_{s}+\varphi_{s}\right) \\
& =\sum_{s=1}^{M} G_{11}(n, s-1 ; \lambda) \varphi_{s} h+\delta \sum_{s=1}^{M} G_{11}(n, s-1 ; \lambda) v_{s} h .
\end{aligned}
$$

Applying equation (12), we get

$$
\begin{aligned}
\delta \sum_{k=1}^{M} G_{11}(n, k-1 ; \lambda) v_{k} h & =\delta \sum_{k=1}^{M} G_{11}(n, k-1 ; \lambda)\left[\sum_{s=0}^{M-1} G_{22}(k, s ; \lambda) \psi_{s} h\right] h \\
& =\sum_{s=0}^{M-1}\left[\delta \sum_{k=1}^{M} G_{11}(n, k-1 ; \lambda) G_{22}(k, s ; \lambda) h\right] \psi_{s} h \\
& =\sum_{s=0}^{M-1} G_{12}(n, s ; \lambda) \psi_{s} h .
\end{aligned}
$$

From the last two formulas it follows that

$$
u_{n}=\sum_{s=1}^{M} G_{11}(n, s-1 ; \lambda) \varphi_{s} h+\sum_{s=0}^{M-1} G_{12}(n, s ; \lambda) \psi_{s} h
$$

Lemma 2.1 is proved.

Lemma 2.2 The following pointwise estimates hold; see equation (7):

$$
\begin{aligned}
& |P|,|Q| \leq \frac{1}{1-r^{M},} \\
& \left|G_{11}(n, s ; \lambda)\right| \leq \frac{1}{a\left(1-r^{M}\right)} \begin{cases}r^{n-s+1}, & 1 \leq s \leq n, \\
r^{M+n-s+1}, & n+1 \leq s \leq n \leq M,\end{cases} \\
& \left|G_{22}(n, s ; \lambda)\right| \leq \frac{1}{a\left(1-r^{M}\right)} \begin{cases}r^{M+s+1-n}, & 0 \leq s \leq n-1, \\
r^{s+1-n}, & n \leq s \leq M-1,\end{cases} \\
& \left|G_{12}(n, s ; \lambda)\right| \leq \frac{1}{a\left(1-r^{M}\right)} \begin{cases}r^{n-s+1}, & 0 \leq s \leq n, \\
r^{s-n+1}, & n+1 \leq s \leq n \leq M-1 .\end{cases}
\end{aligned}
$$

Here $r=\frac{1}{1+\frac{(\delta+\lambda) h}{a}}$. 
Proof It is easy to see that the estimates of equations (14), (15), and (16) follow from the triangle inequality. Applying the triangle inequality, we get

$$
\left|G_{12}(n, s ; \lambda)\right| \leq \delta \sum_{p=1}^{s}\left|G_{11}(n, p-1 ; \lambda)\right|\left|G_{22}(p, s ; \lambda)\right| h .
$$

If $1 \leq s \leq n-1$. Then, using the estimates of equations (14), (15), (16), and inequality (18), we get

$$
\begin{aligned}
\left|G_{12}(n, s ; \lambda)\right| \leq & \delta \sum_{p=1}^{s}\left|G_{11}(n, p-1 ; \lambda)\right|\left|G_{22}(p, s ; \lambda)\right| h \\
& +\delta \sum_{p=s+1}^{n}\left|G_{11}(n, p-1 ; \lambda)\right|\left|G_{22}(p, s ; \lambda)\right| h \\
& +\delta \sum_{p=n+1}^{M}\left|G_{11}(n, p-1 ; \lambda)\right|\left|G_{22}(p, s ; \lambda)\right| h \\
\leq & \frac{\delta}{a^{2}\left(1-r^{M}\right)^{2}}\left[\sum_{p=1}^{s} r^{n-p+1} r^{s+1-p} h+\sum_{p=s+1}^{n} r^{n-p+1} r^{M+s+1-p} h\right. \\
& \left.+\sum_{p=n+1}^{M} r^{M+n-p+1} r^{M+s+1-p} h\right] \\
= & \frac{\delta r^{n-s+2}}{a^{2}\left(1-r^{M}\right)^{2}}\left[\sum_{p=1}^{s} r^{2 s-2 p} h+\sum_{p=s+1}^{n} r^{M+2 s-2 p} h+\sum_{p=n+1}^{M} r^{2 M+2 s-2 p} h\right] \\
= & \frac{\delta h r^{n-s+2}}{a^{2}\left(1-r^{M}\right)^{2}\left(1-r^{2}\right)^{2}}\left[1-r^{2 s}+\left(1-r^{2(n-s)}\right) r^{M+2 s-2 n}+r^{2 s}\left(1-r^{2(M-n)}\right)\right] \\
= & \frac{\delta h r^{n-s+2}\left(1-r^{M}\right)}{a^{2}\left(1-r^{M}\right)^{2}\left(1-r^{2}\right)}\left[1+r^{M+2 s-2 n}\right] \\
= & \frac{\delta h r^{n-s+2}\left(1-r^{M}\right)}{a^{2}\left(1-r^{M}\right)\left(1-r^{2}\right)}\left[1+r^{M+2 s-2 n}\right] \\
= & \frac{\delta h r^{n-s+2}\left(1-r^{M}\right)}{a^{2}\left(1-r^{M}\right) r \frac{\delta+\lambda}{a} h(1+r)}\left[1+r^{M+2 s-2 n}\right] \leq \frac{r^{n-s+1}}{a\left(1-r^{M}\right)} .
\end{aligned}
$$

If $s=n$. Then, using the estimates of equations (14), (15), (16), and inequality (18), we get

$$
\begin{aligned}
\left|G_{12}(n, s ; \lambda)\right| \leq & \delta \sum_{p=1}^{n}\left|G_{11}(n, p-1 ; \lambda)\right|\left|G_{22}(p, s ; \lambda)\right| h \\
& +\delta \sum_{p=n+1}^{M}\left|G_{11}(n, p-1 ; \lambda)\right|\left|G_{22}(p, s ; \lambda)\right| h \\
\leq & \frac{\delta}{a^{2}\left(1-r^{M}\right)^{2}}\left[\sum_{p=1}^{n} r^{n-p+1} r^{s+1-p} h+\sum_{p=n+1}^{M} r^{M+n-p+1} r^{M+s+1-p} h\right] \\
= & \frac{\delta r^{2}}{a^{2}\left(1-r^{M}\right)^{2}}\left[\sum_{p=1}^{n} r^{2 s-2 p} h+\sum_{p=n+1}^{M} r^{2 M+2 s-2 p} h\right]
\end{aligned}
$$




$$
\begin{aligned}
& =\frac{\delta h r^{2}}{a^{2}\left(1-r^{M}\right)^{2}\left(1-r^{2}\right)}\left[1-r^{2 n}+\left(1-r^{2(M-s)}\right) r^{2 s}\right] \\
& =\frac{\delta h r^{2}\left(1-r^{M}\right)}{a^{2}\left(1-r^{M}\right)^{2}\left(1-r^{2}\right)}=\frac{\delta h r^{2}\left(1-r^{M}\right)}{a^{2}\left(1-r^{M}\right) r \frac{\delta+\lambda}{a} h(1+r)} \leq \frac{r}{a\left(1-r^{M}\right)} .
\end{aligned}
$$

Here $n \leq s \leq M$. Then, using the estimates of equations (14), (15), (16), and inequality (18), we get

$$
\begin{aligned}
\left|G_{12}(n, s ; \lambda)\right| \leq & \delta \sum_{p=1}^{n}\left|G_{11}(n, p-1 ; \lambda)\right|\left|G_{22}(p, s ; \lambda)\right| h \\
& +\delta \sum_{p=n+1}^{s}\left|G_{11}(n, p-1 ; \lambda)\right|\left|G_{22}(p, s ; \lambda)\right| h \\
& +\delta \sum_{p=s+1}^{M}\left|G_{11}(n, p-1 ; \lambda)\right|\left|G_{22}(p, s ; \lambda)\right| h \\
\leq & \frac{\delta}{a^{2}\left(1-r^{M}\right)^{2}}\left[\sum_{p=1}^{n} r^{n-p+1} r^{s+1-p} h+\sum_{p=n+1}^{s} r^{s+2-p} r^{M+n-p} h\right. \\
& \left.+\sum_{p=s+1}^{M} r^{s+2-p} r^{M+n-p} h\right] \\
= & \frac{\delta r^{s-n+2}}{a^{2}\left(1-r^{M}\right)^{2}}\left[\sum_{p=1}^{n} r^{2 n-2 p} h+\sum_{p=n+1}^{s} r^{M+2 n-2 p} h+\sum_{p=s+1}^{M} r^{2 M+2 n-2 p} h\right] \\
= & \frac{\delta h r^{s-n+2}}{a^{2}\left(1-r^{M}\right)^{2}\left(1-r^{2}\right)^{2}}\left[1-r^{2 n}+\left(1-r^{2(n-s)}\right) r^{M+2 s-2 n}+r^{2 n}\left(1-r^{2(M-s)}\right)\right] \\
= & \frac{\delta h r^{s-n+2}\left(1-r^{M}\right)}{a^{2}\left(1-r^{M}\right)^{2}\left(1-r^{2}\right)}\left[1+r^{M+2 s-2 n}\right] \\
= & \frac{\delta h r^{s-n+1}\left(1-r^{M}\right)}{a^{2}\left(1-r^{M}\right)\left(1-r^{2}\right)}\left[1+r^{M+2 s-2 n}\right] \\
= & \frac{\delta h r^{s-n+2}}{a^{2}\left(1-r^{M}\right) r \frac{\delta+\lambda}{a} h(1+r)}\left[1+r^{M+2 s-2 n}\right] \leq \frac{r^{s-n+1}}{a\left(1-r^{M}\right)} . \\
&
\end{aligned}
$$

Lemma 2.2 is proved.

Theorem 2.1 The operator $\left(\lambda I+A_{h}^{x}\right)$ has a bounded inverse in $\mathbb{C}_{h}$ for any $\lambda \geq 0$ and the following estimate holds:

$$
\left\|\left(\lambda+A_{h}^{x}\right)^{-1}\right\|_{\mathbb{C}_{h} \rightarrow \mathbb{C}_{h}} \leq \frac{M_{1}}{1+\lambda} .
$$

Proof Using the formula equation (13) and the triangle inequality, we get

$$
\begin{aligned}
\left|u_{n}\right| & \leq \sum_{k=1}^{M} h\left|G_{11}(n, k-1 ; \lambda)\right| \max _{1 \leq k \leq M}\left|\varphi_{k}\right|+\sum_{k=0}^{M-1} h\left|G_{12}(n, k ; \lambda)\right| \max _{0 \leq k \leq M-1}\left|\psi_{k}\right| \\
& \leq\left[\sum_{k=1}^{M} h\left|G_{11}(n, k-1 ; \lambda)\right|+\sum_{k=0}^{M-1} h\left|G_{12}(n, k ; \lambda)\right|\right]\left\|\left(\begin{array}{c}
\varphi \\
\psi
\end{array}\right)^{h}\right\|_{C_{h}[0, l]},
\end{aligned}
$$




$$
\begin{aligned}
\left|v_{n}\right| & \leq \sum_{k=0}^{M-1} h\left|G_{22}(n, k ; \lambda)\right| \max _{0 \leq k \leq M-1}\left|\psi_{k}\right| \\
& \leq \sum_{k=0}^{M-1} h\left|G_{22}(n, k ; \lambda)\right|\left\|\left(\begin{array}{c}
\varphi \\
\psi
\end{array}\right)^{h}\right\|_{C_{h}[0, l]},
\end{aligned}
$$

for any $n=0,1, \ldots, M$. Using the estimate of equation (15), we get

$$
\begin{aligned}
\sum_{k=1}^{M} h\left|G_{11}(n, k-1 ; \lambda)\right| & \leq \frac{r}{a\left(1-r^{M}\right)}\left[\sum_{k=1}^{n} r^{n-k} h+\sum_{k=n+1}^{M} r^{M+n-k} h\right] \\
& =\frac{h r}{a\left(1-r^{M}\right) r \frac{\delta+\lambda}{a} h}\left[1-r^{n}+r^{n}\left(1-r^{M-n}\right)\right]=\frac{1}{\delta+\lambda} .
\end{aligned}
$$

Using the estimate of equation (16), we get

$$
\begin{aligned}
\sum_{k=0}^{M-1} h\left|G_{22}(n, k ; \lambda)\right| & \leq \frac{1}{a\left(1-r^{M}\right)}\left[\sum_{k=0}^{n-1} r^{M+k+1-n} h+\sum_{k=n}^{M-1} r^{k+1-n} h\right] \\
& =\frac{h r}{a\left(1-r^{M}\right) r \frac{\delta+\lambda}{a} h}\left[1-r^{M-n}+r^{M-n}\left(1-r^{n}\right)\right]=\frac{1}{\delta+\lambda}
\end{aligned}
$$

Using the estimate of equation (17), we get

$$
\begin{aligned}
\sum_{k=0}^{M-1} h\left|G_{12}(n, k ; \lambda)\right| & \leq \frac{r}{a\left(1-r^{M}\right)}\left[\sum_{k=0}^{n} r^{n-k} h+\sum_{k=n+1}^{M-1} r^{k-n} h\right] \\
& =\frac{h r}{a\left(1-r^{M}\right) r \frac{\delta+\lambda}{a} h}\left[1-r^{n+1}+r\left(1-r^{M-n-1}\right)\right] \leq \frac{2}{\delta+\lambda} .
\end{aligned}
$$

Therefore,

$$
\begin{aligned}
\max _{1 \leq n \leq M}\left|u_{n}\right| & \leq\left[\sum_{k=1}^{M} h\left|G_{11}(n, k-1 ; \lambda)\right|+\sum_{k=0}^{M-1} h\left|G_{12}(n, k ; \lambda)\right|\right]\left\|\left(\begin{array}{c}
\varphi \\
\psi
\end{array}\right)^{h}\right\|_{C_{h}[0, l]} \\
& \leq \frac{3}{\delta+\lambda}\left\|\left(\begin{array}{c}
\varphi \\
\psi
\end{array}\right)^{h}\right\|_{C_{h}[0, l]}, \\
\max _{0 \leq n \leq M-1}\left|v_{n}\right| & \leq \sum_{k=0}^{M-1} h\left|G_{22}(n, k ; \lambda)\right|\left\|\left(\begin{array}{c}
\varphi \\
\psi
\end{array}\right)^{h}\right\|_{C_{h}[0, l]} \leq \frac{1}{\delta+\lambda}\left\|\left(\begin{array}{c}
\varphi \\
\psi
\end{array}\right)^{h}\right\|_{C_{h}[0, l]} .
\end{aligned}
$$

From this it follows that

$$
\left\|\left(\begin{array}{l}
u \\
v
\end{array}\right)^{h}\right\|_{C_{h}[0, l]} \leq \frac{3}{\delta+\lambda}\left\|\left(\begin{array}{l}
\varphi \\
\psi
\end{array}\right)^{h}\right\|_{C_{h}[0, l]}
$$

Theorem 2.1 is proved. 


\section{The structure of fractional spaces $E_{\alpha}\left(\mathbb{C}_{h}, A_{h}^{x}\right)$ and positivity of $A_{h}^{x}$ in Hölder spaces}

Clearly, the operator $A_{h}^{x}$ and its resolvent $\left(A_{h}^{x}+\lambda\right)^{-1}$ commute. By the definition of the norm in the fractional space $E_{\alpha}=E_{\alpha}\left(\mathbb{C}_{h}, A_{h}^{x}\right)$, we get

$$
\left\|\left(A_{h}^{x}+\lambda\right)^{-1}\right\|_{E_{\alpha} \rightarrow E_{\alpha}} \leq\left\|\left(A_{h}^{x}+\lambda\right)^{-1}\right\|_{\mathbb{C}_{h} \rightarrow \mathbb{C}_{h}}
$$

Thus, from Theorem 2.1 it follows that $A_{h}^{x}$ is a positive operator in the fractional spaces $E_{\alpha}\left(\mathbb{C}_{h}, A_{h}^{x}\right)$. Moreover, we have the following result.

Theorem 3.1 For $\alpha \in(0,1)$, the norms of the spaces $E_{\alpha}\left(\mathbb{C}_{h}, A_{h}^{x}\right)$ and the Hölder space $\stackrel{\circ}{C}_{h}^{\alpha}$ are equivalent uniformly with respect to $h$. Here

$$
\begin{aligned}
\stackrel{\circ}{C}_{h}^{\alpha}= & \left\{\left(\begin{array}{l}
\varphi \\
\psi
\end{array}\right)^{h} \in \mathbb{C}_{h}^{\alpha}:\right. \\
& \left.\varphi_{0}=\gamma \varphi_{M}, 0 \leq \gamma \leq 1, \beta \psi_{0}=\psi_{M}, 0 \leq \beta \leq 1\right\} .
\end{aligned}
$$

Proof For any $\lambda \geq 0$ we have the obvious equality

$$
A_{h}^{x}\left(A_{h}^{x}+\lambda\right)^{-1}\left(\begin{array}{c}
\varphi_{n} \\
\psi_{n}
\end{array}\right)=\left(\begin{array}{l}
\varphi_{n} \\
\psi_{n}
\end{array}\right)-\lambda\left(A_{h}^{x}+\lambda\right)^{-1}\left(\begin{array}{c}
\varphi_{n} \\
\psi_{n}
\end{array}\right) .
$$

By equation (8), we can write

$$
\begin{aligned}
A_{h}^{x}\left(A_{h}^{x}+\lambda\right)^{-1}\left(\begin{array}{c}
\varphi_{n} \\
\psi_{n}
\end{array}\right) & \\
= & \left(\begin{array}{c}
\varphi_{n} \\
\psi_{n}
\end{array}\right)-\lambda \sum_{k=0}^{M} G(n, k ; \lambda) h\left(\begin{array}{c}
\varphi_{n} \\
\psi_{n}
\end{array}\right) \\
= & \left(\begin{array}{c}
{\left[1-\lambda \sum_{k=1}^{n} G_{11}(n, k ; \lambda) h\right] \varphi_{n}-\lambda \sum_{k=n+1}^{M} G_{11}(n, k ; \lambda) h \varphi_{M}} \\
0
\end{array}\right) \\
& +\left(\begin{array}{c}
-\lambda \sum_{k=0}^{M-1} G_{12}(n, k ; \lambda) h \psi_{n} \\
-\lambda \sum_{k=0}^{n-1} G_{22}(n, k ; \lambda) h \psi_{0}+\left[1-\lambda \sum_{k=n}^{M-1} G_{22}(n, k ; \lambda) h\right] \psi_{n}
\end{array}\right) \\
& +\left(\begin{array}{c}
\lambda \sum_{k=1}^{n} G_{11}(n, k ; \lambda)\left(\varphi_{n}-\varphi_{k}\right) h+\lambda \sum_{k=n+1}^{M} G_{11}(n, k ; \lambda)\left(\varphi_{M}-\varphi_{k}\right) h \\
0
\end{array}\right) \\
& +\left(\begin{array}{c}
\lambda \sum_{k=0}^{M-1} G_{12}(n, k ; \lambda)\left(\psi_{n}-\psi_{k}\right) h \\
\lambda \sum_{k=0}^{n-1} G_{12}(n, k ; \lambda)\left(\psi_{0}-\psi_{k}\right) h+\lambda \sum_{k=n}^{M-1} G_{22}(n, k ; \lambda)\left(\psi_{n}-\psi_{k}\right) h
\end{array}\right) .
\end{aligned}
$$

Applying equation (21) and the following obvious equalities:

$$
\begin{aligned}
1-\lambda \sum_{k=1}^{n} G_{11}(n, k ; \lambda) h & =1-Q \lambda \sum_{k=1}^{n} U(n, s-1 ; \lambda) \frac{h}{a_{s}} \\
& =1-Q \frac{\lambda}{\delta+\lambda} \sum_{k=1}^{n}(U(n, s-1 ; \lambda)-U(n, s ; \lambda))
\end{aligned}
$$




$$
\begin{aligned}
& =1-Q \frac{\lambda}{\delta+\lambda}(1-U(n, 0 ; \lambda)) \\
& =\frac{\delta}{\delta+\lambda} Q-\gamma Q U(M, 0 ; \lambda)+\frac{\lambda}{\delta+\lambda} Q U(n, 0 ; \lambda), \\
& -\lambda \sum_{k=n+1}^{M} G_{11}(n, k ; \lambda)=-Q \lambda \sum_{k=n+1}^{M} \frac{\gamma}{a_{s}} U(n, s-1 ; \lambda) U(M, 0 ; \lambda) \\
& =-\frac{\lambda}{\delta+\lambda} Q \sum_{k=n+1}^{M} \gamma U(M, 0 ; \lambda)(U(n, s-1 ; \lambda)-U(n, s ; \lambda)) \\
& =-\frac{\lambda}{\delta+\lambda} \gamma Q U(M, 0 ; \lambda)(U(n, 1 ; \lambda)-U(n, M ; \lambda)) \\
& =-\frac{\lambda}{\delta+\lambda} \gamma Q[U(M, 0 ; \lambda) U(n, 1 ; \lambda)-U(n, 0 ; \lambda)], \\
& 1-\lambda \sum_{s=n}^{M-1} G_{22}(n, k ; \lambda) h=1-P \lambda \sum_{s=n}^{M-1} \frac{1}{a_{s+1}} U(s+1, n ; \lambda) h \\
& =1-P \frac{\lambda}{\delta+\lambda} \sum_{s=n}^{M-1}[U(s, n ; \lambda)-U(s+1, n ; \lambda)] h \\
& =1-P \frac{\lambda}{\delta+\lambda}[1-U(M, n ; \lambda)] h \\
& =P \frac{\delta}{\delta+\lambda}-P \beta U(M, 0 ; \lambda)+P \frac{\lambda}{\delta+\lambda} U(M, n ; \lambda), \\
& -\lambda \sum_{s=0}^{n-1} G_{22}(n, k ; \lambda) h=-P \lambda \sum_{s=0}^{n-1} \frac{\beta}{a_{s+1}} U(M, n ; \lambda) U(s+1,0 ; \lambda) h \\
& -\lambda \beta \sum_{s=0}^{n-1} P U(M, n ; \lambda) \frac{1}{\delta+\lambda}[U(s, n ; \lambda)-U(s+1, n ; \lambda)] h \\
& -\lambda \beta P \frac{1}{\delta+\lambda} U(M, n ; \lambda)[U(0, n ; \lambda)-1] h, \\
& -\lambda \sum_{s=0}^{M-1} G_{12}(n, s ; \lambda) h=-\lambda \sum_{s=0}^{M-1} \delta h \sum_{k=1}^{M} G_{11}(n, k-1 ; \lambda) G_{22}(k, s ; \lambda) h \\
& =-\lambda \sum_{k=1}^{M}\left[\sum_{s=0}^{k-1} \delta h G_{11}(n, k-1 ; \lambda) G_{22}(k, s ; \lambda) h\right. \\
& \left.+\sum_{s=k}^{M-1} \delta h G_{11}(n, k-1 ; \lambda) G_{22}(k, s ; \lambda) h\right] \\
& =-\lambda \sum_{k=1}^{n+1} \sum_{s=0}^{k-1} \delta h G_{11}(n, k-1 ; \lambda) G_{22}(k, s ; \lambda) h \\
& -\lambda \sum_{k=n+2}^{M} \sum_{s=0}^{k-1} \delta h G_{11}(n, k-1 ; \lambda) G_{22}(k, s ; \lambda) h \\
& =-\lambda \sum_{k=1}^{n+1} \sum_{s=k}^{M-1} \delta h G_{11}(n, k-1 ; \lambda) G_{22}(k, s ; \lambda) h
\end{aligned}
$$




$$
\begin{aligned}
& -\lambda \sum_{k=n+2}^{M} \sum_{s=k}^{M-1} \delta h G_{11}(n, k-1 ; \lambda) G_{22}(k, s ; \lambda) h \\
& =-\lambda \sum_{k=1}^{n+1} \sum_{s=0}^{k-1} \delta h U(n, k-2 ; \lambda) \frac{1}{a_{k-1}} \frac{\beta}{a_{s+1}} \\
& \times U(M, k ; \lambda) U(s+1,0 ; \lambda) h \\
& =-\lambda \sum_{k=n+2}^{M} \sum_{s=0}^{k-1} \delta h \gamma U(M, 0 ; \lambda) U(n, k-2 ; \lambda) \frac{1}{a_{k-1}} \frac{\beta}{a_{s+1}} \\
& \times U(M, k ; \lambda) U(s+1,0 ; \lambda) h \\
& =-\lambda \sum_{k=1}^{n+1} \sum_{s=k}^{M-1} \delta h U(n, k-2 ; \lambda) \frac{1}{a_{k-1}} \frac{1}{a_{s+1}} U(s+1, k ; \lambda) h \\
& =-\lambda \sum_{k=n+2}^{M} \sum_{s=k}^{M-1} \delta h \gamma U(M, 0 ; \lambda) U(n, k-2 ; \lambda) \frac{1}{a_{k-1}} \frac{1}{a_{s+1}} U(s+1, k ; \lambda) h \\
& =-\lambda \sum_{k=1}^{n+1} \sum_{s=0}^{k-1} \delta h U(n, k-2 ; \lambda) \frac{1}{a_{k-1}} U(M, k ; \lambda) \beta \frac{1}{\delta+\lambda} \\
& \times[U(s, 0 ; \lambda)-U(s+1,0 ; \lambda)] \\
& -\lambda \sum_{k=n+2}^{M} \sum_{s=0}^{k-1} \delta h \gamma U(M, 0 ; \lambda) U(n, k-2 ; \lambda) \frac{1}{a_{k-1}} \beta \frac{1}{\delta+\lambda} \\
& \times[U(s, 0 ; \lambda)-U(s+1,0 ; \lambda)] \\
& -\lambda \sum_{k=1}^{n+1} \sum_{s=k}^{M-1} \delta h U(n, k-2 ; \lambda) \frac{1}{a_{k-1}} \frac{1}{\delta+\lambda} \\
& \times[U(s, 0 ; \lambda)-U(s+1,0 ; \lambda)] \\
& -\lambda \sum_{k=n+2}^{M} \sum_{s=k}^{M-1} \delta h \gamma U(M, 0 ; \lambda) U(n, k-2 ; \lambda) \frac{1}{a_{k-1}} \beta \frac{1}{\delta+\lambda} \\
& \times[U(s, 0 ; \lambda)-U(s+1,0 ; \lambda)] \\
& =-\lambda \sum_{k=1}^{n+1} \delta h U(n, k-2 ; \lambda) \frac{1}{a_{k-1}} U(M, k ; \lambda) \beta \frac{1}{\delta+\lambda}[1-U(k, 0 ; \lambda)] \\
& -\lambda \sum_{k=n+2}^{M} \delta h \gamma U(M, 0 ; \lambda) U(n, k-2 ; \lambda) \frac{1}{a_{k-1}} \beta \frac{1}{\delta+\lambda}[1-U(k, 0 ; \lambda)] \\
& -\lambda \sum_{k=1}^{n+1} \delta h U(n, k-2 ; \lambda) \frac{1}{a_{k-1}} \frac{1}{\delta+\lambda}[U(k, 0 ; \lambda)-U(M, 0 ; \lambda)] \\
& -\lambda \sum_{k=n+2}^{M} \delta h \gamma U(M, 0 ; \lambda) U(n, k-2 ; \lambda) \frac{1}{a_{k-1}} \frac{1}{\delta+\lambda} \\
& \times[U(k, 0 ; \lambda)-U(M, 0 ; \lambda)],
\end{aligned}
$$


and using the nonlocal boundary conditions

$$
\varphi_{0}=\gamma \varphi_{M}, \quad \beta \psi_{0}=\psi_{M},
$$

we get

$$
\begin{aligned}
& A_{h}^{x}\left(\lambda+A_{h}^{x}\right)^{-1}\left(\begin{array}{l}
\varphi_{n} \\
\psi_{n}
\end{array}\right) \\
& =\left(\begin{array}{c}
{\left[\frac{\delta}{\delta+\lambda} Q-\gamma Q U(M, 0 ; \lambda)\right] \varphi_{n}+Q \frac{\lambda}{\delta+\lambda} U(n, 0 ; \lambda)\left(\varphi_{n}-\varphi_{0}\right)} \\
0
\end{array}\right) \\
& =\left(\begin{array}{c}
\frac{\lambda}{\delta+\lambda} \gamma Q U(M, 0 ; \lambda) U(n, 1 ; \lambda) \varphi_{M} \\
-\lambda \beta P U(M, n ; \lambda) \frac{1}{\delta+\lambda} U(0, n ; \lambda) \psi_{0}
\end{array}\right) \\
& +\left(\begin{array}{c}
\left\{-\lambda \sum_{k=1}^{n+1} \delta h U(n, k-2 ; \lambda) \frac{1}{a_{k-1}} U(M, k ; \lambda) \beta \frac{1}{\delta+\lambda}[1-U(k, 0 ; \lambda)]\right\} \psi_{n} \\
{\left[P \frac{\delta}{\delta+\lambda}-P \beta U(M, 0 ; \lambda)\right] \psi_{n}+P \frac{\lambda}{\delta+\lambda} U(M, n ; \lambda)\left(\psi_{n}-\psi_{M}\right)}
\end{array}\right) \\
& +\left(\begin{array}{c}
-\lambda \sum_{k=1}^{n+1} \delta h U(n, k-2 ; \lambda) \frac{1}{a_{k-1}} U(M, k ; \lambda) \beta \frac{1}{\delta+\lambda}[1-U(k, 0 ; \lambda)] \psi_{n} \\
0
\end{array}\right) \\
& +\left(\begin{array}{c}
-\lambda \sum_{k=n+2}^{M} \delta h \gamma U(M, 0 ; \lambda) U(n, k-2 ; \lambda) \frac{1}{a_{k-1}} \beta \frac{1}{\delta+\lambda}[1-U(k, 0 ; \lambda)] \psi_{n} \\
0
\end{array}\right) \\
& +\left(\begin{array}{c}
\left.-\lambda \sum_{k=1}^{n+1} \delta h U(n, k-2 ; \lambda) \frac{1}{a_{k-1}} \frac{1}{\delta+\lambda}[U(k, 0 ; \lambda)-U(M, 0 ; \lambda)] \psi_{n}\right) \\
0
\end{array}\right) \\
& +\left(\begin{array}{c}
-\lambda \sum_{k=n+2}^{M} \delta h \gamma U(M, 0 ; \lambda) U(n, k-2 ; \lambda) \frac{1}{a_{k-1}} \beta \frac{1}{\delta+\lambda}[U(k, 0 ; \lambda)-U(M, 0 ; \lambda)] \psi_{n} \\
0
\end{array}\right) \\
& +\left(\begin{array}{c}
\lambda \sum_{k=1}^{n+1} G_{11}(n, k ; \lambda)\left(\varphi_{n}-\varphi_{k}\right) h+\lambda \sum_{k=n+1}^{M} G_{11}(n, k ; \lambda)\left(\varphi_{M}-\varphi_{k}\right) h \\
0
\end{array}\right) \\
& +\left(\begin{array}{c}
\lambda \sum_{k=0}^{M-1} G_{12}(n, k ; \lambda)\left(\psi_{n}-\psi_{k}\right) h \\
\lambda \sum_{k=0}^{n-1} G_{22}(n, k ; \lambda)\left(\psi_{0}-\psi_{k}\right) h+\lambda \sum_{k=n}^{M-1} G_{22}(n, k ; \lambda)\left(\psi_{n}-\psi_{k}\right) h
\end{array}\right) .
\end{aligned}
$$

Using this formula, the triangle inequality and the definition of spaces $E_{\alpha}\left(\mathbf{C}[0, l]_{h}, A_{h}^{x}\right)$ and $\mathbf{C}^{\alpha}[0, l]_{h}$, we get

$$
\begin{aligned}
& \left\|\lambda^{\alpha} A_{h}^{x}\left(A_{h}^{x}+\lambda\right)^{-1}\left(\begin{array}{c}
\varphi \\
\psi
\end{array}\right)^{h}\right\| \|_{\mathbb{C}\left[0, l_{h}\right.} \\
& \leq \max _{1 \leq n \leq M}\left[\frac{\lambda^{\alpha} \delta}{\delta+\lambda}|Q|+\gamma|Q| \lambda^{\alpha}|U(M, 0 ; \lambda)|+\frac{\lambda^{1+\alpha}}{\delta+\lambda} \gamma|Q||U(M, 0 ; \lambda)||U(n, 1 ; \lambda)|\right] \\
& \quad \times \max _{0 \leq n \leq M}\left|\psi_{n}\right| \\
& \quad+\max _{1 \leq n \leq M} \frac{\lambda^{1+\alpha}}{\delta+\lambda}|Q|(n h)^{\alpha}|U(n, 0 ; \lambda)| \sup _{1 \leq n \leq M} \frac{\left|\varphi_{n}-\varphi_{0}\right|}{(n h)^{\alpha}} \\
& \quad+\max _{1 \leq n \leq M}\left[\frac{\lambda^{\alpha} \delta}{\delta+\lambda}|P|+\beta|P| \lambda^{\alpha}|U(M, 0 ; \lambda)|+\frac{\lambda^{1+\alpha}}{\delta+\lambda} \beta|P||U(M, n ; \lambda)||U(0, n ; \lambda)|\right]
\end{aligned}
$$




$$
\begin{aligned}
& \times \max _{0 \leq n \leq M}\left|\psi_{n}\right|+\max _{1 \leq n \leq M} \frac{\lambda^{1+\alpha}}{\delta+\lambda}|P|((M-n) h)^{\alpha}|U(M, n ; \lambda)| \\
& \times \sup _{0 \leq n \leq M-1} \frac{\left|\psi_{n}-\psi_{M}\right|}{((M-n) h)^{\alpha}} \\
& +\max _{1 \leq n \leq M} \lambda^{1+\alpha} \sum_{k=1}^{n+1} \delta h|U(n, k-2 ; \lambda)| \frac{1}{a_{k-1}}|U(M, k ; \lambda)| \\
& \times \beta \frac{1}{\delta+\lambda}|1-U(k, 0 ; \lambda)| \max _{0 \leq n \leq M}\left|\psi_{n}\right| \\
& +\max _{1 \leq n \leq M} \lambda^{1+\alpha} \sum_{k=n+2}^{M} \delta h \gamma|U(M, 0 ; \lambda)||U(n, k-2 ; \lambda)| \frac{1}{a_{k-1}} \beta \frac{1}{\delta+\lambda}|1-U(k, 0 ; \lambda)| \\
& \times \max _{0 \leq n \leq M}\left|\psi_{n}\right| \\
& +\max _{1 \leq n \leq M} \lambda^{1+\alpha} \sum_{k=1}^{n+1} \delta h|U(n, k-2 ; \lambda)| \frac{1}{a_{k-1}} \frac{1}{\delta+\lambda}|U(k, 0 ; \lambda)-U(M, k ; \lambda)| \max _{0 \leq n \leq M}\left|\psi_{n}\right| \\
& +\max _{1 \leq n \leq M} \lambda^{1+\alpha} \sum_{k=n+2}^{M} \delta h \gamma|U(M, 0 ; \lambda)||U(n, k-2 ; \lambda)| \frac{1}{a_{k-1}} \\
& \times \frac{1}{\delta+\lambda}|U(k, 0 ; \lambda)-U(M, 0 ; \lambda)| \max _{0 \leq n \leq M}\left|\psi_{n}\right| \\
& +\max _{1 \leq n \leq M} \lambda^{1+\alpha} \sum_{k=1}^{n+1}((n-k) h)^{\alpha}\left|G_{11}(n, k ; \lambda)\right| h \sup _{1 \leq k \leq n \leq M} \frac{\left|\varphi_{n}-\varphi_{k}\right|}{((n-k) h)^{\alpha}} \\
& +\max _{1 \leq n \leq M} \lambda^{1+\alpha} \sum_{k=n+1}^{M}((M-n) h)^{\alpha}\left|G_{11}(n, k ; \lambda)\right| h \sup _{1 \leq n \leq M-1} \frac{\left|\varphi_{M}-\varphi_{k}\right|}{((M-n) h)^{\alpha}} \\
& +\max _{1 \leq n \leq M} \lambda^{1+\alpha} \sum_{k=0}^{M-1}(|k-n| h)^{\alpha}\left|G_{12}(n, k ; \lambda)\right| h \sup _{\substack{1 \leq k, n \leq M, k \neq n}} \frac{\left|\psi_{n}-\psi_{k}\right|}{(|n-k| h)^{\alpha}} \\
& +\max _{1 \leq n \leq M} \lambda^{1+\alpha} \sum_{k=0}^{n-1}(k h)^{\alpha}\left|G_{22}(n, k ; \lambda)\right| h \sup _{1 \leq k \leq M} \frac{\left|\psi_{k}-\psi_{0}\right|}{(k h)^{\alpha}} \\
& +\max _{1 \leq n \leq M} \lambda^{1+\alpha} \sum_{k=n+1}^{M-1}((k-n) h)^{\alpha}\left|G_{22}(n, k ; \lambda)\right| h \sup _{1 \leq n \leq k \leq M-1} \frac{\left|\psi_{n}-\psi_{k}\right|}{((k-n) h)^{\alpha}} \\
& \leq J\left\|\left(\begin{array}{c}
\varphi \\
\psi
\end{array}\right)^{h}\right\|_{\mathbb{C}_{h}^{\alpha}}
\end{aligned}
$$

Here

$$
\begin{aligned}
J= & \frac{\lambda^{\alpha} \delta}{\delta+\lambda}|Q|+\gamma|Q| \lambda^{\alpha}|U(M, 0 ; \lambda)| \\
& +\max _{1 \leq n \leq M}\left\{\frac{\lambda^{1+\alpha}}{\delta+\lambda} \gamma|Q||U(M, 0 ; \lambda)||U(n, 1 ; \lambda)|+\frac{\lambda^{1+\alpha}}{\delta+\lambda}|Q|(n h)^{\alpha}|U(n, 0 ; \lambda)|\right. \\
& \times \frac{\lambda^{\alpha} \delta}{\delta+\lambda}|P|+\beta|P| \lambda^{\alpha}|U(M, 0 ; \lambda)|+\frac{\lambda^{1+\alpha}}{\delta+\lambda} \beta|P||U(M, n ; \lambda)||U(0, n ; \lambda)|
\end{aligned}
$$




$$
\begin{aligned}
& +\frac{\lambda^{1+\alpha}}{\delta+\lambda}|P|((M-n) \tau)^{\alpha}|U(M, n ; \lambda)| \\
& +\lambda^{1+\alpha} \sum_{k=1}^{n+1} \delta h|U(n, k-2 ; \lambda)| \frac{1}{a_{k-1}}|U(M, k ; \lambda)| \beta \frac{1}{\delta+\lambda}|1-U(k, 0 ; \lambda)| \\
& +\lambda^{1+\alpha} \sum_{k=n+2}^{M} \delta h \gamma|U(M, 0 ; \lambda)||U(n, k-2 ; \lambda)| \frac{1}{a_{k-1}} \beta \frac{1}{\delta+\lambda}|1-U(k, 0 ; \lambda)| \\
& +\lambda^{1+\alpha} \sum_{k=1}^{n+1} \delta h|U(n, k-2 ; \lambda)| \frac{1}{a_{k-1}} \frac{1}{\delta+\lambda}|U(k, 0 ; \lambda)-U(M, k ; \lambda)|+\lambda^{1+\alpha} \\
& \times \sum_{k=n+2}^{M} \delta h \gamma|U(M, 0 ; \lambda)||U(n, k-2 ; \lambda)| \frac{1}{a_{k-1}} \frac{1}{\delta+\lambda}|U(k, 0 ; \lambda)-U(M, 0 ; \lambda)| \\
& +\lambda^{1+\alpha} \sum_{k=1}^{n+1}((n-k) \tau)^{\alpha}\left|G_{11}(n, k ; \lambda)\right| h+\lambda^{1+\alpha} \sum_{k=n+1}^{M}((M-n) \tau)^{\alpha}\left|G_{11}(n, k ; \lambda)\right| h \\
& +\lambda^{1+\alpha} \sum_{k=0}^{M-1}(|k-n| \tau)^{\alpha}\left|G_{12}(n, k ; \lambda)\right| h+\lambda^{1+\alpha} \sum_{k=0}^{n-1}(k \tau)^{\alpha}\left|G_{22}(n, k ; \lambda)\right| h \\
& \left.+\lambda^{1+\alpha} \sum_{k=n+1}^{M-1}((k-n) \tau)^{\alpha}\left|G_{22}(n, k ; \lambda)\right| h\right\} .
\end{aligned}
$$

Using the estimates

$$
\frac{\lambda^{\alpha} \delta^{1-\alpha}}{\delta+\lambda} \leq 1, \quad \frac{\lambda^{1+\alpha} \delta^{1-\alpha}}{(\delta+\lambda)^{2}} \leq 1
$$

and the estimates of equations (14), (15), (16), and (17), we get

$$
\begin{aligned}
J \leq & \max _{1 \leq n \leq M}\left\{\frac{\lambda^{\alpha} \delta}{\delta+\lambda} \frac{1}{1-r^{M}}+\frac{1}{1-r^{M}} \lambda^{\alpha}\left(1+\frac{(\delta+\lambda) h}{a}\right)^{-M}\right. \\
& +\frac{\lambda^{1+\alpha}}{\delta+\lambda} \frac{1}{1-r^{M}}\left(1+\frac{(\delta+\lambda) h}{a}\right)^{-M-n+1}+\frac{\lambda^{1+\alpha}}{\delta+\lambda} \frac{1}{1-r^{M}}(n \tau)^{\alpha}\left(1+\frac{(\delta+\lambda) h}{a}\right)^{-n} \\
& +\frac{\lambda^{\alpha} \delta}{\delta+\lambda} \frac{1}{1-r^{M}}+\frac{1}{1-r^{M}} \lambda^{\alpha}\left(1+\frac{(\delta+\lambda) h}{a}\right)^{-M}+\frac{\lambda^{1+\alpha}}{\delta+\lambda} \frac{1}{1-r^{M}}\left(1+\frac{(\delta+\lambda) h}{a}\right)^{-M} \\
& +\frac{\lambda^{1+\alpha}}{\delta+\lambda} \frac{1}{1-r^{M}}((M-n) h)^{\alpha}\left(1+\frac{(\delta+\lambda) h}{a}\right)^{-M+n} \\
& +\lambda^{1+\alpha} \sum_{k=1}^{n+1} \delta h r^{n-k+2+M-k} \frac{1}{a} \frac{1}{\delta+\lambda}+\lambda^{1+\alpha} \sum_{k=n+2}^{M} \delta h r^{M+n-k+2} \frac{1}{a} \frac{1}{\delta+\lambda} \\
& +\lambda^{1+\alpha} \sum_{k=1}^{n+1} \delta h r^{n-k+2} \frac{1}{a} \frac{1}{\delta+\lambda}+\lambda^{1+\alpha} \sum_{k=n+2}^{M} \delta h r^{M+n-k+2} \frac{1}{a} \frac{1}{\delta+\lambda} \\
& +\lambda^{1+\alpha} \sum_{k=1}^{n+1}((n-k) h)^{\alpha} r^{n-k+2} \frac{1}{a} h+\lambda^{1+\alpha} \sum_{k=n+1}^{M}((M-k) h)^{\alpha} r^{M-k+2} \frac{1}{a} h
\end{aligned}
$$




$$
\begin{aligned}
& +\lambda^{1+\alpha} \sum_{k=0}^{M-1}(|k-n| h)^{\alpha} r^{|n-k|+1} \frac{1}{a} h+\lambda^{1+\alpha} \sum_{k=0}^{n-1}(k h)^{\alpha} r^{k+1} \frac{1}{a} h \\
& \left.+\lambda^{1+\alpha} \sum_{k=n+1}^{M-1}((k-n) h)^{\alpha} r^{k-n+2} \frac{1}{a} h\right\} \leq M(a, \delta) .
\end{aligned}
$$

Then

$$
\left\|\lambda^{\alpha} A_{h}^{x}\left(A_{h}^{x}+\lambda\right)^{-1}\left(\begin{array}{c}
\varphi \\
\psi
\end{array}\right)^{h}\right\|_{\mathbb{C}_{h}} \leq M(a, \delta)\left\|\left(\begin{array}{l}
\varphi \\
\psi
\end{array}\right)^{h}\right\|_{\mathbb{C}_{h}^{\alpha}}
$$

for any $\lambda \geq 0$. This means that

$$
\left\|\left(\begin{array}{c}
\varphi \\
\psi
\end{array}\right)^{h}\right\|_{E_{\alpha}\left(C_{h}, A_{h}^{\alpha}\right)} \leq M(a, \delta)\left\|\left(\begin{array}{c}
\varphi \\
\psi
\end{array}\right)^{h}\right\|_{\mathbb{C}_{h}^{\alpha}} .
$$

Let us prove the opposite inequality. For any positive operator $A_{h}^{x}$ we can write

$$
\left(\begin{array}{l}
f \\
g
\end{array}\right)=\int_{0}^{\infty} A_{h}^{x}\left(\lambda+A_{h}^{x}\right)^{-2}\left(\begin{array}{l}
f \\
g
\end{array}\right) d \lambda
$$

From the relation and formula (21) it follows that

$$
\begin{aligned}
\left(\begin{array}{c}
\varphi_{n} \\
\psi_{n}
\end{array}\right)= & \int_{0}^{\infty}\left(\lambda+A_{h}^{x}\right)^{-1} A_{h}^{x}\left(\lambda+A_{h}^{x}\right)^{-1}\left(\begin{array}{c}
\varphi_{n} \\
\psi_{h}
\end{array}\right) d \lambda \\
= & \int_{0}^{\infty} \sum_{k=0}^{M} G(n, k ; \lambda) h A_{h}^{x}\left(\lambda+A_{h}^{x}\right)^{-1}\left(\begin{array}{c}
\varphi_{k} \\
\psi_{k}
\end{array}\right) d \lambda \\
= & \int_{0}^{\infty} \sum_{s=1}^{M} G_{11}(n, s ; \lambda) h A_{h}^{x}\left(\lambda+A_{h}^{x}\right)^{-1}\left(\begin{array}{c}
\varphi_{s} \\
0
\end{array}\right) d \lambda \\
& +\int_{0}^{\infty} \sum_{s=0}^{M-1} G_{12}(n, s ; \lambda) h A_{h}^{x}\left(\lambda+A_{h}^{x}\right)^{-1}\left(\begin{array}{c}
0 \\
\psi_{s}
\end{array}\right) d \lambda \\
& +\int_{0}^{\infty} \sum_{s=0}^{M-1} G_{22}(n, s ; \lambda) h A_{h}^{x}\left(\lambda+A_{h}^{x}\right)^{-1}\left(\begin{array}{c}
0 \\
\psi_{s}
\end{array}\right) d \lambda .
\end{aligned}
$$

Consequently,

$$
\begin{aligned}
\left(\begin{array}{l}
\varphi_{n+m} \\
\psi_{n+m}
\end{array}\right) & =\left(\begin{array}{l}
\varphi_{n} \\
\psi_{n}
\end{array}\right) \\
& =\int_{0}^{\infty} \sum_{k=0}^{M-1}(G(n+m, k ; \lambda)-G(n, k ; \lambda)) h A_{h}^{x}\left(\lambda+A_{h}^{x}\right)^{-1}\left(\begin{array}{c}
\varphi_{k} \\
\psi_{k}
\end{array}\right) d \lambda,
\end{aligned}
$$


whence

$$
\begin{aligned}
\left|\varphi_{n+m}-\varphi_{n}\right| \leq & \int_{0}^{\infty} \sum_{s=1}^{M} \lambda^{-\alpha}\left|G_{11}(n+m, s ; \lambda)-G_{11}(n, s ; \lambda)\right| h d \lambda\left\|\left(\begin{array}{c}
\varphi \\
\psi
\end{array}\right)^{h}\right\|_{E_{\alpha}\left(C_{h}, A_{h}^{x}\right)} \\
& +\int_{0}^{\infty} \sum_{s=0}^{M-1} \lambda^{-\alpha}\left|G_{12}(n+m, s ; \lambda)-G_{12}(n, s ; \lambda)\right| h d \lambda\left\|\left(\begin{array}{c}
\varphi \\
\psi
\end{array}\right)^{h}\right\|_{E_{\alpha}\left(C_{h}, A_{h}^{x}\right)}, \\
\left|\psi_{n+m}-\psi_{n}\right| \leq & \int_{0}^{\infty} \sum_{s=0}^{M-1} \lambda^{-\alpha}\left|G_{22}(n+m, s ; \lambda)-G_{22}(n, s ; \lambda)\right| h d \lambda\left\|\left(\begin{array}{c}
\varphi \\
\psi
\end{array}\right)^{h}\right\|_{E_{\alpha}\left(C_{h}, A_{h}^{x}\right)} .
\end{aligned}
$$

Let

$$
\begin{aligned}
P= & (m h)^{-\alpha} \int_{0}^{\infty} \sum_{s=1}^{M} \lambda^{-\alpha}\left|G_{11}(n+m, s ; \lambda)-G_{11}(n, s ; \lambda)\right| h d \lambda \\
& +(m h)^{-\alpha} \int_{0}^{\infty} \sum_{s=0}^{M-1} \lambda^{-\alpha}\left|G_{12}(n+m, s ; \lambda)-G_{12}(n, s ; \lambda)\right| h d \lambda \\
& +(m h)^{-\alpha} \int_{0}^{\infty} \sum_{s=0}^{M-1} \lambda^{-\alpha}\left|G_{22}(n+m, s ; \lambda)-G_{22}(n, s ; \lambda)\right| h d \lambda .
\end{aligned}
$$

Then for any $n+m, n \in\{0,1, \ldots, M\}$, we have

$$
(m h)^{-\alpha}\left|\varphi_{n+m}-\varphi_{n}\right|+(m h)^{-\alpha}\left|\psi_{n+m}-\psi_{n}\right| \leq P\left\|\left(\begin{array}{c}
\varphi \\
\psi
\end{array}\right)^{h}\right\|_{E_{\alpha}\left(C\left[0, l_{h}, A_{h}^{x}\right)\right.} .
$$

Now let us estimate $P=P_{1}+P_{2}+P_{3}$, where

$$
\begin{aligned}
& P_{1}=(m h)^{-\alpha} \int_{0}^{\infty} \sum_{s=1}^{M} \lambda^{-\alpha}\left|G_{11}(n+m, s ; \lambda)-G_{11}(n, s ; \lambda)\right| h d \lambda, \\
& P_{2}=(m h)^{-\alpha} \int_{0}^{\infty} \sum_{s=0}^{M-1} \lambda^{-\alpha}\left|G_{12}(n+m, s ; \lambda)-G_{12}(n, s ; \lambda)\right| h d \lambda, \\
& P_{3}=(m h)^{-\alpha} \int_{0}^{\infty} \sum_{s=0}^{M-1} \lambda^{-\alpha}\left|G_{22}(n+m, s ; \lambda)-G_{22}(n, s ; \lambda)\right| h d \lambda .
\end{aligned}
$$

Note that it suffices to consider the case when $0 \leq m h \leq \frac{1}{2}$. Applying the scheme of the paper [33] and using equations (8), (9), (10), (11), and the estimates of equations (14), (15), (16), and (17), we can establish the following estimate:

$$
P_{r} \leq \frac{M_{6}(a, \delta)}{\alpha(1-\alpha)}
$$

for $r=1,2,3$. Applying the triangle inequality and the estimate of equation (22), we get

$$
P \leq \frac{M_{7}(a, \delta)}{\alpha(1-\alpha)}
$$


Thus for any $n+m, n \in\{0,1, \ldots, M\}$ we have

$$
|m h|^{-\alpha}\left|\varphi_{n+m}-\varphi_{n}\right|+|m h|^{-\alpha}\left|\psi_{n+m}-\psi_{n}\right| \leq \frac{M_{8}(a, \delta)}{\alpha(1-\alpha)}\left\|\left(\begin{array}{c}
\varphi \\
\psi
\end{array}\right)^{h}\right\|_{E_{\alpha}\left(C_{h}, A_{h}\right)} .
$$

This means that the following inequality holds:

$$
\left\|\left(\begin{array}{c}
\varphi \\
\psi
\end{array}\right)^{h}\right\|_{C_{h}^{\alpha}} \leq \frac{M_{8}(a, \delta)}{\alpha(1-\alpha)}\left\|\left(\begin{array}{c}
\varphi \\
\psi
\end{array}\right)^{h}\right\|_{E_{\alpha}\left(C_{h}, A_{h}\right)} .
$$

Theorem 3.1 is proved.

Since the $A_{h}^{x}$ is a positive operator in the fractional spaces $E_{\alpha}\left(\mathbb{C}_{h}, A_{h}^{x}\right)$, from the result of Theorem 3.1 it follows that it is also a positive operator in the Hölder space $\stackrel{\circ}{C}_{h}^{\alpha}$. Namely, we have the following.

Theorem 3.2 The operator $\left(\lambda I+A_{h}^{x}\right)$ has a bounded inverse in $\stackrel{\circ}{C}_{h}^{\alpha}$ uniformly with respect to $h$ for any $\lambda \geq 0$ and the following estimate holds:

$$
\left\|\left(\lambda I+A_{h}^{x}\right)^{-1}\right\|_{\mathbb{C}_{h}^{\alpha} \rightarrow \stackrel{\mathbb{C}}{h}_{h}^{\alpha}} \leq \frac{M_{8}(a, \delta)}{\alpha(1-\alpha)} \frac{M_{1}}{1+\lambda}
$$

\section{Applications}

In this section we consider the application of results of Sections 2 and 3. For a positive operator $A$ in $E$ the following result was established in papers [36, 37].

Theorem 4.1 Let $A$ be a positive operator in E. Then it obeys the following estimate:

$$
\left\|\mathbf{R}_{q, q-1}^{k}(\tau A)\right\|_{E \rightarrow E} \leq M_{1}, \quad 1 \leq k \leq N, N \tau=T
$$

where $M_{1}$ does not depend on $\tau$ and $k$. Here $\mathbf{R}_{q, q-1}(z)$ is the Padé approximation of $\exp (-z)$ near $z=0$.

For a numerical solution of the initial-boundary value problem (1) the following difference scheme is presented:

$$
\left\{\begin{array}{l}
\frac{u_{n}^{k}-u_{n}^{k-1}}{\tau}+a\left(x_{n}\right) \frac{u_{n}^{k}-u_{n-1}^{k}}{h}+\delta\left(u_{n}^{k}-v_{n}^{k}\right)=f_{1, n}^{k}, \quad f_{1, n}^{k}=f_{1}\left(t_{k}, x_{n}\right), \\
t_{k}=k \tau, x_{n}=n h, 1 \leq k \leq N, N \tau=T, 1 \leq n \leq M, M h=l, \\
\frac{v_{n}^{k}-v_{n}^{k-1}}{\tau}-a\left(x_{n+1}\right) \frac{v_{n+1}^{k}-v_{n}^{k}}{h}+\delta v_{n}^{k}=f_{2, n}^{k}, \quad f_{2, n}^{k}=f_{2}\left(t_{k}, x_{n}\right), \\
t_{k}=k \tau, x_{n}=n h, 1 \leq k \leq N, N \tau=T, 0 \leq n \leq M-1, M h=l, \\
u_{0}^{k}=\gamma u_{M}^{k}, \quad 0 \leq \gamma \leq 1, \quad \beta v_{0}^{k}=v_{M}^{k}, \quad 0 \leq \beta \leq 1,0 \leq k \leq N, \\
u_{n}^{0}=u_{0}\left(x_{n}\right), \quad v_{n}^{0}=v_{0}\left(x_{n}\right), \quad x_{n}=n h, 0 \leq n \leq M, M h=l .
\end{array}\right.
$$

We introduce the Banach space $\mathbb{C}\left([0, T]_{\tau}, E\right)$ of all continuous abstract mesh vector functions

$$
u^{\tau}=\left\{u^{k}\right\}_{k=1}^{N}=\left\{\left(\begin{array}{l}
u_{1, n}^{k} \\
u_{2, n}^{k}
\end{array}\right)^{h}\right\}_{k=1}^{N}
$$


defined on $[0, T]_{\tau}=\left\{t_{k}=k \tau, 1 \leq k \leq N, N \tau=T\right\}$ with values in $E$, equipped with the norm

$$
\left\|u^{\tau}\right\|_{\mathbb{C}\left([0, T]_{\tau}, E\right)}=\max _{1 \leq k \leq N}\left\|\left\{u_{1, n}^{k}\right\}_{n=1}^{M}\right\|_{E}+\max _{1 \leq k \leq N}\left\|\left\{u_{2, n}^{k}\right\}_{n=1}^{M}\right\|_{E}
$$

Note that the problem (24) can be written in the form of the abstract Cauchy problem

$$
\begin{aligned}
& \left\{\left(\begin{array}{c}
\frac{u^{k}-u^{k-1}}{\tau} \\
\frac{v^{k}-v^{k-1}}{\tau}
\end{array}\right)^{h}\right\}_{k=1}^{N}+A_{h}^{x}\left\{\left(\begin{array}{c}
u^{k} \\
v^{k}
\end{array}\right)^{h}\right\}_{k=1}^{N}=\left\{\left(\begin{array}{l}
f_{1}^{k} \\
f_{2}
\end{array}\right)^{h}\right\}_{k=1}^{N}, \quad 1 \leq k \leq N, \\
& \left(\begin{array}{c}
u_{0} \\
v_{0}
\end{array}\right)=\left(\begin{array}{c}
u_{n}^{0} \\
v_{n-1}^{0}
\end{array}\right)_{n=1}^{M}
\end{aligned}
$$

in a Banach space $E=\mathbb{C}_{h}$ with a positive operator $A_{h}^{x}$ defined by (5). Here $\left\{\left(\begin{array}{l}f_{1}^{k} \\ f_{2}^{k}\end{array}\right)^{h}\right\}_{k=1}^{N}=$ $\left\{\left(\begin{array}{c}f_{1, n}^{k} \\ f_{2, n-1}^{k}\end{array}\right)_{n=1}^{M}\right\}_{k=1}^{N}$ is the given abstract vector function defined on $[0, T]_{\tau}$ with values in $E$, $\left(\begin{array}{c}u_{0} \\ v_{0}\end{array}\right)=\left(\begin{array}{c}u_{n}^{0} \\ v_{n-1}^{0}\end{array}\right)_{n=1}^{M}$ is the element of $D\left(A_{h}^{x}\right)$. It is well known that (see, for example [3]) the formula

$$
\left(\begin{array}{l}
u^{k} \\
v^{k}
\end{array}\right)=\left(I+\tau A_{h}^{x}\right)^{-k}\left(\begin{array}{l}
u_{0} \\
v_{0}
\end{array}\right)+\sum_{j=1}^{k}\left(I+\tau A_{h}^{x}\right)^{-k+j-1}\left(\begin{array}{l}
f_{1}^{j} \\
f_{2}^{j}
\end{array}\right) \tau
$$

gives a solution of the problem $(25)$ in $\mathbb{C}\left([0, T]_{\tau}, E\right)$.

Theorem 4.2 For the solution of the problem (25) the stability inequality holds:

$$
\left\|\left\{\left(\begin{array}{c}
u^{k} \\
v^{k}
\end{array}\right)\right\}_{k=1}^{N}\right\|_{\mathbb{C}\left([0, T]_{\tau}, E\right)} \leq M(a, \delta)\left[\left\|\left(\begin{array}{c}
u_{0} \\
v_{0}
\end{array}\right)\right\|_{E}+\left\|\left\{\left(\begin{array}{l}
f_{1}^{k} \\
f_{2}^{k}
\end{array}\right)\right\}_{k=1}^{N}\right\|_{\mathbb{C}\left([0, T]_{\tau}, E\right)}\right] .
$$

The proof of Theorem 4.2 is based on the positivity of the operator $A_{h}^{x}$, equation (26) and the estimate of equation (23).

Applying the results of Theorems 2.1 and 4.2, we get the following theorem.

Theorem 4.3 The solution of the problem (24) satisfies the following estimate:

$$
\begin{aligned}
& \max _{1 \leq k \leq N} \max _{1 \leq n \leq M}\left|u_{n}^{k}\right|+\max _{1 \leq k \leq N} \max _{0 \leq n \leq M-1}\left|v_{n}^{k}\right| \\
& \quad \leq M(a, \delta)\left[\max _{1 \leq n \leq M}\left|u_{n}^{0}\right|+\max _{0 \leq n \leq M-1}\left|v_{n}^{0}\right|+\max _{1 \leq k \leq N} \max _{1 \leq n \leq M}\left|f_{1, n}^{k}\right|+\max _{1 \leq k \leq N} \max _{0 \leq n \leq M-1}\left|f_{2, n}^{k}\right|\right] .
\end{aligned}
$$

Applying results of Theorems 3.2, 4.1, and 4.2, we get the following theorem.

\section{Theorem 4.4 Assume that}

$$
\begin{array}{ll}
f_{1,0}^{k}=\gamma f_{1, M}^{k}, & 0 \leq \gamma \leq 1, \\
\beta f_{2,0}^{k}=f_{2, M}^{k}, & 0 \leq \beta \leq 1,1 \leq k \leq N .
\end{array}
$$


Then the solution of the problem (24) satisfies the following estimate:

$$
\begin{aligned}
\max _{1 \leq k \leq N} & \left(\max _{1 \leq n \leq M}\left|u_{n}^{k}\right|+\sup _{1 \leq n<n+m \leq N} \frac{\left|u_{n+m}^{k}-u_{n}^{k}\right|}{(m \tau)^{\alpha}}\right) \\
& +\max _{1 \leq k \leq N}\left(\max _{0 \leq n \leq M-1}\left|v_{n}^{k}\right|+\sup _{0 \leq n<n+m \leq M-1} \frac{\left|v_{n+m}^{k}-v_{n}^{k}\right|}{(m \tau)^{\alpha}}\right) \\
\leq & M(a, \delta, \alpha)\left[\max _{1 \leq n \leq M}\left|u_{n}^{0}\right|+\sup _{1 \leq n<n+m \leq N} \frac{\left|u_{n+m}^{0}-u_{n}^{0}\right|}{(m \tau)^{\alpha}}\right. \\
& +\max _{0 \leq n \leq M-1}\left|v_{n}^{0}\right|+\sup _{0 \leq n<n+m \leq M-1} \frac{\left|v_{n+m}^{0}-v_{n}^{0}\right|}{(m \tau)^{\alpha}} \\
& +\max _{1 \leq k \leq N}\left(\max _{1 \leq n \leq M}\left|f_{1, n}^{k}\right|+\sup _{1 \leq n<n+m \leq N} \frac{\left|f_{1, n+m}^{k}-f_{1, n}^{k}\right|}{(m \tau)^{\alpha}}\right) \\
& \left.+\max _{1 \leq k \leq N}\left(\max _{0 \leq n \leq M-1}\left|f_{2, n}^{k}\right|+\sup _{0 \leq n<n+m \leq M-1} \frac{\left|f_{2, n+m}^{k}-f_{2, n}^{k}\right|}{(m \tau)^{\alpha}}\right)\right] .
\end{aligned}
$$

Finally, one has not been able to obtain a sharp estimate for the constants figuring in the stability estimates. Therefore, our interest in the present paper is studying the difference scheme equation (24) by numerical experiments. Applying this difference scheme, the numerical method is proposed in the following section for the numerical solution of the hyperbolic system of equations with nonlocal boundary conditions. The method is illustrated by a numerical example.

\section{Numerical results}

For the numerical result, the initial value problem

$$
\left\{\begin{array}{l}
\frac{\partial u}{\partial t}=-\frac{\partial u}{\partial x}-(u-v)+(1+2 \pi) \cos (2 \pi x+t), \quad 0<t<1,0<x<1, \\
\frac{\partial v}{\partial t}=\frac{\partial v}{\partial x}-v+(1-2 \pi) \cos (2 \pi x+t)+\sin (2 \pi x+t), \quad 0<t<1,0<x<1, \\
u(t, 0)=u(t, 1), \quad v(t, 0)=v(t, 1), \quad 0 \leq t \leq 1, \\
u(0, x)=v(0, x)=\sin 2 \pi x, \quad 0 \leq x \leq 1
\end{array}\right.
$$

for the hyperbolic system of equations with nonlocal boundary conditions is considered. Applying the difference scheme equation (24), we obtain

$$
\left\{\begin{array}{l}
\frac{u_{n}^{k}-u_{n}^{k-1}}{\tau}=-\frac{u_{n}^{k}-u_{n-1}^{k}}{h}-u_{n}^{k}+v_{n}^{k}+\varphi_{n}^{k}, \\
1 \leq n \leq M, 1 \leq k \leq N, M h=1, N \tau=1, \\
\frac{v_{n}^{k}-v_{n}^{k-1}}{\tau}=\frac{v_{n+1}^{k}-v_{n}^{k}}{h}-v_{n}^{k}+\psi_{n}^{k}, \\
\quad 0 \leq n \leq M-1,1 \leq k \leq N, \\
u_{0}^{k}=u_{M}^{k}, \quad v_{0}^{k}=v_{M}^{k}, \quad 0 \leq k \leq N, \\
u_{n}^{0}=v_{n}^{0}=\sin (2 \pi n h), \quad 0 \leq n \leq M,
\end{array}\right.
$$

where

$$
\left\{\begin{array}{l}
\varphi_{n}^{k}=(1+2 \pi) \cos (2 \pi n h+k \tau), \\
\psi_{n}^{k}=(1-2 \pi) \cos (2 \pi n h+k \tau)+\sin (2 \pi n h+k \tau) .
\end{array}\right.
$$


We get the system of equations in the matrix form

$$
\begin{aligned}
& J_{1} v^{k}=B v^{k-1}+\varphi^{k}, \quad 1 \leq k \leq N, v^{0}=\psi, \\
& J_{2} u^{k}=B u^{k-1}+v^{k}+\psi^{k}, \quad 1 \leq k \leq N, u^{0}=\varphi,
\end{aligned}
$$

where

$$
\begin{aligned}
& v^{k}=\left(\begin{array}{c}
v_{0}^{k} \\
v_{1}^{k} \\
\vdots \\
v_{M}^{k}
\end{array}\right), \quad \psi=\left(\begin{array}{c}
0 \\
\sin 2 \pi h \\
\sin 4 \pi h \\
\vdots \\
\sin 2 M \pi h
\end{array}\right) \\
& u_{k}=\left(\begin{array}{c}
u_{0}^{k} \\
u_{1}^{k} \\
\vdots \\
u_{M}^{k}
\end{array}\right), \quad \varphi=\left(\begin{array}{c}
0 \\
\sin 2 \pi h \\
\sin 4 \pi h \\
\vdots \\
\sin 2 M \pi h
\end{array}\right) \text {, } \\
& J_{1}=\left(\begin{array}{ccccccc}
a & b & 0 & 0 & \cdots & 0 & 0 \\
0 & a & b & 0 & \cdots & 0 & 0 \\
0 & 0 & a & b & \cdots & 0 & 0 \\
\cdot & . & . & . & \cdots & . & . \\
0 & 0 & 0 & 0 & \cdots & a & b \\
1 & 0 & 0 & 0 & \cdots & 0 & -1
\end{array}\right)_{(N+1) \times(M+1)} \\
& B=\left(\begin{array}{ccccc}
c & 0 & 0 & \cdots & 0 \\
0 & c & 0 & \cdots & 0 \\
\cdot & \cdot & . & \cdots & . \\
0 & 0 & 0 & 0 & c \\
0 & 0 & 0 & 0 & 0
\end{array}\right)_{(M+1) \times(M+1)} \\
& J_{2}=\left(\begin{array}{ccccccc}
a & f & 0 & 0 & \cdots & 0 & 0 \\
0 & a & f & 0 & \cdots & 0 & 0 \\
0 & 0 & a & f & \cdots & 0 & 0 \\
\cdot & \cdot & \cdot & \cdot & \cdots & . & \cdot \\
0 & 0 & 0 & 0 & \cdots & a & f \\
1 & 0 & 0 & 0 & \cdots & 0 & -1
\end{array}\right)_{(M+1) \times(M+1)} \\
& a=\frac{1}{\tau}-\frac{1}{h}+1, f=-\frac{1}{h}, b=\frac{1}{h}, c=\frac{1}{\tau}, \\
& \varphi^{k}=\left(\begin{array}{c}
\varphi_{0}^{k} \\
\varphi_{1}^{k} \\
\vdots \\
\varphi_{M-1}^{k} \\
0
\end{array}\right), \quad \psi^{k}=\left(\begin{array}{c}
\psi_{0}^{k} \\
\psi_{1}^{k} \\
\vdots \\
\psi_{M-1}^{k} \\
0
\end{array}\right)
\end{aligned}
$$


Table 1 Difference scheme

\begin{tabular}{lll}
\hline & $\boldsymbol{M}=\boldsymbol{N}=\mathbf{5 0}$ & $\boldsymbol{M}=\boldsymbol{N}=\mathbf{1 0 0}$ \\
\hline Comparison of errors for $u$ & 0.0444 & 0.0226 \\
Comparison of errors for $v$ & 0.0875 & 0.0436 \\
\hline
\end{tabular}

Thus, we have the first-order difference equation with respect to $k$ matrix coefficients. To solve this difference equation we have the following procedure:

$$
\left\{\begin{array}{l}
v^{k}=J_{1}^{-1} B v^{k-1}+J_{1}^{-1} \varphi^{k}, \quad 1 \leq k \leq N, v^{0}=\psi \\
u^{k}=J_{2}^{-1} B u^{k-1}+J_{2}^{-1} v^{k}+J_{2}^{-1} \psi^{k}, \quad 1 \leq k \leq N, u^{0}=\varphi
\end{array}\right.
$$

For their comparison, the errors are computed by

$$
\begin{aligned}
& E_{u}=\max _{1 \leq k \leq N-1,1 \leq n \leq M-1}\left|u\left(t_{k}, x_{n}\right)-u_{n}^{k}\right|, \\
& E_{v}=\max _{1 \leq k \leq N-1,1 \leq n \leq M-1}\left|v\left(t_{k}, x_{n}\right)-v_{n}^{k}\right|
\end{aligned}
$$

of the numerical solutions. The numerical solutions are recorded for different values of $N=M ; u_{n}^{k}, v_{n}^{k}$ represent the numerical solutions of these difference schemes at $\left(t_{k}, x_{n}\right)$. The errors are given in Table 1 for $N=M=50$, and $N=M=100$, respectively.

\section{Conclusion}

In the present study, the finite-difference method for the initial-boundary value problem for the hyperbolic system of equations with nonlocal boundary conditions is studied. The positivity of the difference analogy of the space operator generated by this problem in the space with maximum norm is established. The structure interpolation spaces generated by this difference operator is investigated. The positivity of this difference operator in Hölder spaces is established. In practice, stability estimates for the solution of the difference scheme for the hyperbolic system of equations with nonlocal boundary conditions are obtained. A numerical example is applied. Moreover, applying this approach we can construct the stable difference schemes for numerical solutions of the initial-boundary value problem

$$
\left\{\begin{array}{l}
\frac{\partial u(t, x)}{\partial t}+a(x) \frac{\partial u(t, x)}{\partial x}+\delta(u(t, x)-v(t, x))=f_{1}(t, x ; u(t, x), v(t, x)) \\
\quad 0<x<l, 0<t<T \\
\frac{\partial v(t, x)}{\partial t}-a(x) \frac{\partial v(t, x)}{\partial x}+\delta v(t, x)=f_{2}(t, x ; v(t, x)) \\
\quad 0<x<l, 0<t<T \\
u(t, 0)=\gamma u(t, l), \quad 0 \leq \gamma \leq 1 \\
\beta v(t, 0)=v(t, l), \quad 0 \leq \beta \leq 1,0 \leq t \leq T \\
u(0, x)=u_{0}(x), \quad v(0, x)=v_{0}(x), \quad 0 \leq x \leq l
\end{array}\right.
$$

for the hyperbolic system of semilinear equations with nonlocal boundary conditions. Of course, convergence estimates for the solution of these difference schemes can be obtained. 


\section{Competing interests}

The authors declare that they have no competing interests.

\section{Authors' contributions}

Each of the authors read and approved the final version of the manuscript.

\section{Acknowledgements}

We would like to thank the reviewers whose careful reading, helpful suggestions, and valuable comments helped us to improve the manuscript.

\section{Received: 12 October 2013 Accepted: 26 December 2013 Published: 20 Jan 2014}

\section{References}

1. Fattorini, HO: Second Order Linear Differential Equations in Banach Spaces. North-Holland, Amsterdam (1985)

2. Goldstein, JA: Semigroups of Linear Operators and Applications. Oxford Mathematical Monographs. Clarendon, New York (1985)

3. Krein, SG: Linear Differential Equations in Banach Space. Nauka, Moscow (1966) (in Russian). English transl.: Linear Differential Equations in Banach space. Translations of Mathematical Monographs, vol. 23. Am. Math. Soc., Providence (1968)

4. Pogorelenko, V, Sobolevskii, PE: The 'counter-example' to W. Littman counter-example of $L_{p}$-energetic inequality for wave equation. Funct. Differ. Equ. 4(1-2), 165-172 (1997)

5. Sobolevskii, PE: On the equations of the second order with a small parameter at the highest derivatives. Usp. Mat. Nauk 19(6), 217-219 (1964) (in Russian)

6. Sobolevskii, PE, Semenov, S: On some approach to investigation of singular hyperbolic equations. Dokl. Akad. Nauk SSSR 270(1), 555-558 (1983) (in Russian)

7. Vasilev, W, Krein, SG, Piskarev, S: Operator semigroups, cosine operator functions, and linear differential equations. In: Mathematical Analysis, vol. 28 (in Russian). Itogi Nauki i Tekhniki, vol. 204, pp. 87-202. Akad. Nauk SSSR Vsesoyuz. Inst. Nauchn. i Tekhn. Inform., Moscow (1990). (Translated in J. Soviet Math. 54(4), 1042-1129 (1991))

8. Ashyralyev, A, Fattorini, $\mathrm{HO}$ : On uniform difference schemes for second-order singular pertubation problems in Banach spaces. SIAM J. Math. Anal. 23(1), 29-54 (1992)

9. Ashyraliyev, M: A note on the stability of the integral-differential equation of the hyperbolic type in a Hilbert space. Numer. Funct. Anal. Optim. 29(7-8), 750-769 (2008)

10. Yurtsever, A, Prenov, R: On stability estimates for method of line for first order system of differential equations of hyperbolic type. In: Ashyralyev, A, Yurtsever, A (eds.) Some Problems of Applied Mathematics, pp. 198-205. Fatih University Publications, Istanbul (2000)

11. Ashyralyev, A, Yildirim, O: A note on the second order of accuracy stable difference schemes for the nonlocal boundary value hyperbolic problem. Abstr. Appl. Anal. 2012, Article ID 846582 (2012)

12. Ashyralyev, A, Aggez, N: Finite difference method for hyperbolic equations with the nonlocal integral condition. Discrete Dyn. Nat. Soc. 2011, Article ID 562385 (2011)

13. Ashyralyev, A, Sobolevskii, PE: Two new approaches for construction of the high order of accuracy difference schemes for hyperbolic differential equations. Discrete Dyn. Nat. Soc. 2005(2), 183-213 (2005)

14. Ashyralyev, A, Koksal, ME, Agarwal, RP: A difference scheme for Cauchy problem for the hyperbolic equation with self-adjoint operator. Math. Comput. Model. 52(1-2), 409-424 (2010)

15. Aggez, N, Ashyralyyeva, M: Numerical solution of stochastic hyperbolic equations. Abstr. Appl. Anal. 2012, Article ID $824819(2012)$

16. Ashyralyev, A, Akat, M: An approximation of stochastic hyperbolic equations. In: AIP Conference Proceedings, vol. 1389, pp. 625-628 (2011)

17. Amanov, D, Ashyralyev, A: Initial-boundary value problem for fractional partial differential equations of higher order. Abstr. Appl. Anal. 2012, Article ID 973102 (2012)

18. Ashyralyev, A, Dal, F: Finite difference and iteration methods for fractional hyperbolic partial differential equations with the Neumann condition. Discrete Dyn. Nat. Soc. 2012, Article ID 434976 (2012)

19. Ashyralyev, A, Yildirim, O: On multipoint nonlocal boundary value problems hyperbolic differential and difference equations. Taiwan. J. Math. 14(1), 165-194 (2010)

20. Soltanov, H: A note on the Goursat problem for a multidimensional hyperbolic equation. Contemp. Anal. Appl. Math. 1(2), 98-106 (2013)

21. Selitskii, AM: The space of initial data for the second boundary-value problem for parabolic differential-difference equation. Contemp. Anal. Appl. Math. 1(1), 34-41 (2013)

22. Selitskii, AM: The space of initial data for the Robin boundary-value problem for parabolic differential-difference equation. Contemp. Anal. Appl. Math. 1(2), 91-97 (2013)

23. Agarwal, R, Bohner, M, Shakhmurov, VB: Maximal regular boundary value problems in Banach-valued weighted spaces. Bound. Value Probl. 1, 9-42 (2005)

24. Shakhmurov, VB: Coercive boundary value problems for regular degenerate differential-operator equations. J. Math. Anal. Appl. 292(2), 605-620 (2004)

25. Ashyralyev, A, Sobolevskii, PE: Well-posedness of parabolic difference equations. In: Operator Theory Advances and Applications. Birkhäuser, Basel (1994)

26. Rassias, JM, Karimov, ET: Boundary-value problems with non-local condition for degenerate parabolic equations. Contemp. Anal. Appl. Math. 1(1), 42-48 (2013)

27. Ashyralyev, A, Sobolevskii, PE: New difference schemes for partial differential equations. In: Operator Theory Advances and Applications. Birkhäuser, Basel (2004)

28. Dehghan, M: On the numerical solution of the diffusion equation with a nonlocal boundary condition. Math. Probl. Eng. 2003(2), 81-92 (2003)

29. Cannon, JR, Perez Esteva, S, van der Hoek, J: A Galerkin procedure for the diffusion equation subject to the specification of mass. SIAM J. Numer. Anal. 24(3), 499-515 (1987) 
30. Gordeziani, N, Natani, P, Ricci, PE: Finite-difference methods for solution of nonlocal boundary value problems. Comput. Math. Appl. 50, 1333-1344 (2005)

31. Dautray, R, Lions, JL: Analyse Mathematique et Calcul Numerique Pour les Sciences et les Techniques, vols. 1-11. Masson, Paris (1988)

32. Ashyralyev, A, Ozdemir, Y: Stability of difference schemes for hyperbolic-parabolic equations. Comput. Math. Appl. 50(8-9), 1443-1476 (2005)

33. Ashyralyev, A, Prenov, R: The hyperbolic system of equations with nonlocal boundary conditions. TWMS J. Appl. Eng. Math. 2(2), 154-178 (2012)

34. Godunov, SK: Numerical Methods of Solution of the Equation of Gas Dynamics. NSU, Novosibirsk (1962) (in Russian)

35. Tikhonov, AN, Samarskii, AA: Equations of Mathematical Physics. Nauka, Moscow (1977) (in Russian)

36. Hersch, R, Kato, T: High accuracy stable difference schemes for well-posed initial value problems. SIAM J. Numer. Anal. 19(3), 599-603 (1982)

37. Brenner, $\mathrm{P}$, Crouzeix, M, Thomee, V: Single step methods for inhomogeneous linear differential equations in Banach space. RAIRO Anal. Numér. 16(1), 5-26 (1982)

10.1186/1687-1847-2014-26

Cite this article as: Ashyralyev and Prenov: Finite-difference method for the hyperbolic system of equations with nonlocal boundary conditions. Advances in Difference Equations 2014, 2014:26

\section{Submit your manuscript to a SpringerOpen ${ }^{\odot}$ journal and benefit from:}

- Convenient online submission

- Rigorous peer review

- Immediate publication on acceptance

- Open access: articles freely available online

- High visibility within the field

- Retaining the copyright to your article 